\title{
The disease burden of bronchiectasis in comparison with chronic obstructive pulmonary disease: a national database study in Korea
}

\author{
Bumhee Yang' ${ }^{1 \#}$, Hayoung Choi ${ }^{2 \#}$, Jun Hyeok Lim ${ }^{3}$, Hye Yun Park ${ }^{4}$, Danbee Kang ${ }^{5,6}$, Juhee Cho ${ }^{5,6,7}$, \\ Jae Seung Lee ${ }^{8}$, Sei Won Lee ${ }^{8}$, Yeon-Mok Oh$^{8}$, Ji-Yong Moon', Sang-Heon Kim ${ }^{9}$, Tae-Hyung Kim?', \\ Jang Won Sohn', Ho Joo Yoon', Hyun Lee'
}

${ }^{1}$ Division of Pulmonology, Center of Lung Cancer, National Cancer Center, Goyang, Korea; ${ }^{2}$ Division of Pulmonary, Allergy, and Critical Care Medicine, Department of Internal Medicine, Hallym University Kangnam Sacred Heart Hospital, Seoul, Korea; ${ }^{3}$ Division of Pulmonology, Department of Internal Medicine, Inha University Hospital, Inha University School of Medicine, Incheon, Korea; ${ }^{4}$ Division of Pulmonary and Critical Care Medicine, Department of Medicine, Samsung Medical Center, Sungkyunkwan University School of Medicine, Seoul, Korea; ${ }^{5}$ Center for Clinical Epidemiology, Samsung Medical Center, Seoul, Korea; ${ }^{6}$ Department of Clinical Research Design and Evaluation, SAIHST, Sungkyunkwan University, Seoul, Korea; ${ }^{7}$ Department of Epidemiology and Welch Center for Prevention, Epidemiology, and Clinical Research, Johns Hopkins University Bloomberg School of Public Health, Baltimore, Maryland, USA; ${ }^{8}$ Department of Pulmonary and Critical Care Medicine and Clinical Research Center for Chronic Obstructive Airway Diseases, Asan Medical Center, University of Ulsan College of Medicine, Seoul, Korea; ${ }^{9}$ Department of Internal Medicine, Hanyang University College of Medicine, Seoul, Korea

Contributions: (I) Conception and design: B Yang, H Choi, H Lee; (II) Administrative support: H Choi, HJ Yoon, JW Sohn, SH Kim, H Lee; (III) Provision of study materials or patients: B Yang, H Choi, JH Lim, H Lee; (IV) Collection and assembly of data: B Yang, H Choi, HY Park, D Kang, J Cho, H Lee; (V) Data analysis and interpretation: B Yang, H Choi, JS Lee, SW Lee, YM Oh, JY Moon, TH Kim, H Lee; (VI) Manuscript writing: All authors; (VII) Final approval of manuscript: All authors.

\#These authors contributed equally to this work.

Correspondence to: Hyun Lee. Division of Pulmonary Medicine and Allergy, Department of Internal Medicine, Hanyang University College of Medicine, 222-1, Wangsimni-ro, Seongdong-gu, Seoul 04763, Korea. Email: namuhanayeyo@naver.com; namuhanayeyo@hanyang.ac.kr.

Background: The prevalence and disease burden of bronchiectasis in comparison to those of chronic obstructive pulmonary disease (COPD) have not been well elucidated using a nationally representative database.

Methods: We compared respiratory symptoms, physical activity, quality of life, and socioeconomic status in subjects with bronchiectasis versus those with COPD or control subjects participating in the Korea National Health and Nutrition Examination Survey 2007-2009. Participants were classified as physician-diagnosed bronchiectasis, COPD, and control (those without COPD or bronchiectasis).

Results: The prevalence of bronchiectasis in subjects aged 40 years or older was $0.8 \%$. Compared to COPD subjects, bronchiectasis subjects were more likely to be younger (mean 59.0 years, $\mathrm{P}<0.001$ ), female (47.6\%, $\mathrm{P}<0.001)$, and never-smoker $(50.1 \%, \mathrm{P}<0.001)$ and have had history of pulmonary tuberculosis $(40.5 \%, \mathrm{P}<0.001)$ and osteoporosis $(19.1 \%, \mathrm{P}=0.025)$. However, as in COPD subjects, bronchiectasis subjects had low family income $(\mathrm{P}<0.001)$ and the proportion of subjects working as manager/professional/office workers $(6.4 \%, \mathrm{P}<0.001)$ was smaller than that of control subjects. After adjusting for covariables, compared to control, bronchiectasis subjects but not COPD subjects were more likely to have respiratory symptoms [adjusted odds ratio $(\mathrm{OR})=7.96,95 \%$ confidence interval (CI): 2.10-30.12], limitation in physical activity (adjusted OR =9.43, 95\% CI: 1.06-83.79), and low family income (adjusted OR =3.61, 95\% CI: 1.75-7.47).

Conclusions: The prevalence of bronchiectasis in subjects at least 40 years of age was $0.8 \%$ in Korea. Despite large number of young patients and low prevalence of smoking history, respiratory symptoms, limitation in physical activity, and low family income were significant burden in bronchiectasis subjects. 
Keywords: Bronchiectasis; prevalence; disease burden; chronic obstructive pulmonary disease (COPD)

Submitted Aug 18, 2019. Accepted for publication Nov 05, 2019.

doi: $10.21037 /$ atm.2019.11.55

View this article at: http://dx.doi.org/10.21037/atm.2019.11.55

\section{Introduction}

Non-cystic fibrosis (CF) patients with bronchiectasis have a chronic respiratory disease characterized by permanent dilatation of bronchi and chronic respiratory symptoms such as cough, sputum, and dyspnea (1). Bronchiectasis has been regarded as an orphan disease in developed countries. However, recent UK data showed that the prevalence of bronchiectasis had been consistently increasing, up to about 490 men and 570 women per 100,000 persons, during the last decade (2). In the US, the annual increase in the prevalence of bronchiectasis has been reported to be approximately $8-9 \%(3,4)$. In addition, these results have drawn attention to the substantial healthcare burden $(2,5)$. In Asia, bronchiectasis is a commonly encountered disease in general practice, and the prevalence of bronchiectasis in Asia is expected to be higher compared to developed countries (6), with about $1.2 \%$ of prevalence estimates in subjects aged 40 years or older in China between 2002-2004 in an urban population-based, cross-sectional survey (7) and about $0.5 \%$ in subjects aged 20 years or older in Korea between 2012-2017 in a representative sample data (8).

Subjects with bronchiectasis often suffer from respiratory symptoms (1), decreased physical activity $(9,10)$, impaired quality of life (11-13), and comorbidities (14). In addition, bronchiectasis is associated with markedly increased healthcare costs and mortality $(5,14)$. Although these studies demonstrated the considerable symptomatic burden of bronchiectasis, it remains a neglected respiratory disease with little interest from experts. Moreover, as clinical characteristics of bronchiectasis were evaluated mainly in cohort studies, unfortunately, the disease burden of bronchiectasis in terms of symptoms, quality of life, and socioeconomic status have not been well elucidated in comparison with other chronic respiratory disease. From this view, the comparison of bronchiectasis with other common chronic respiratory diseases, such as chronic obstructive pulmonary disease (COPD), would be helpful to elucidate the disease burden of bronchiectasis.

Therefore, we investigated the prevalence of bronchiectasis and evaluated the disease burden of this disease in terms of symptoms, quality of life, and socioeconomic status by comparing subjects with bronchiectasis and those with COPD or controls using national representative data in Korea. In addition, we evaluated factors associated with bronchiectasis.

\section{Methods}

\section{Participants}

We used data from the Korea National Health and Nutrition Examination Survey (NHANES) 2007 to 2009, which is a national representative survey collected by the Korean Ministry of Health and Welfare. Health-related questionnaires, health examinations, and spirometric measurements were used in this study. Participants were excluded if they had missing weight variable information $(\mathrm{n}=2,489)$. We restricted our analysis to participants over 40 years old with available spirometric results $(n=6,934)$. According to the presence of bronchiectasis and COPD [pre-bronchodilator forced expiratory volume in 1 second $\left(\mathrm{FEV}_{1}\right)$ /forced vital capacity $\left.(\mathrm{FVC})<0.7\right]$, participants were classified into three groups: participants with bronchiectasis, those with COPD, and control (participants without COPD or bronchiectasis). Those who had both COPD and bronchiectasis were classified in the bronchiectasis group (Figure 1).

\section{Measurements}

Information on age, sex, body mass index, smoking history, physical activity, occupation, the EuroQoL five dimensions questionnaire (EQ-5D) index values, which range between 0 (worst imaginable health state) and 1 (best imaginable health state); and spirometric results were obtained from the Korea NHANES database. Spirometry was performed according to the recommendations of the American Thoracic Society and European Respiratory Society (15). Absolute values of $\mathrm{FEV}_{1}$ and $\mathrm{FVC}$ were obtained, and the percentages of predicted values (\% predicted) for $\mathrm{FEV}_{1}$ and FVC were calculated using the reference equation obtained on analysis of a representative Korean sample (16). COPD was defined as pre-bronchodilator $\mathrm{FEV}_{1} / \mathrm{FVC}$ $<0.7$. Type of occupation was categorized according to the major groups of the $6^{\text {th }}$ Korean Standard Classification 


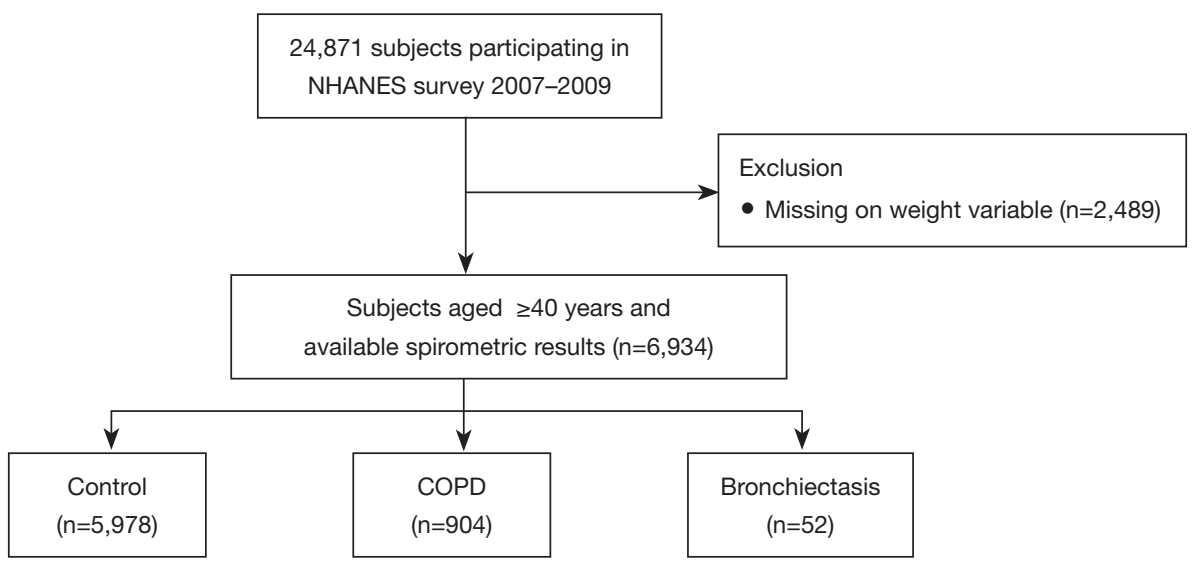

Figure 1 Flow chart of the study population.

of occupation into five groups: manager, professional, or office worker; service or sales worker; agriculture or fishery worker; skilled labor or machine operator; and manual laborer (17). Comorbidities of bronchiectasis, asthma, pulmonary tuberculosis, cardiovascular disease, osteoporosis, osteoarthritis or rheumatoid arthritis, and depression were self-reported based on previous physician diagnosis (18). Hypertension was defined as a self-reported physician diagnosis, the use of antihypertensive medication, a systolic blood pressure $\geq 140 \mathrm{mmHg}$, or a diastolic blood pressure $\geq 90 \mathrm{mmHg}$ (19). Dyslipidemia was defined as a self-reported physician diagnosis, the use of lipid-lowering medication, total cholesterol $\geq 240 \mathrm{mg} / \mathrm{dL}$, or fasting triglyceride $\geq 200 \mathrm{mg} / \mathrm{dL}$ $(20,21)$. Diabetes mellitus (DM) was defined as a physician diagnosis based on the use of glucose-lowering medications or a fasting plasma glucose level $\geq 126 \mathrm{mg} / \mathrm{dL}$ (22).

\section{Outcomes}

We compared respiratory symptoms, physical activity, quality of life, and socioeconomic status among subjects with bronchiectasis versus those with COPD or controls participating in the Korea NHEANES 2007-2009. We further evaluated factors associated with bronchiectasis among the participants.

\section{Statistical analysis}

All analyses were performed using svy commands in STATA 15.1 version (StataCorp LP, College Station, TX, USA) to account for the complex sampling design. For each variable, we calculated its prevalence and $95 \%$ confidence interval
(CI) by the group. To evaluate factors associated with COPD or bronchiectasis, univariable and multivariable bivariate logistic regression analyses were performed. We adjusted for age, sex, body mass index, smoking history, family income, education level, physician-diagnosed asthma, previous pulmonary tuberculosis, and $\mathrm{FEV}_{1}$.

\section{Results}

\section{Baseline characteristics}

The overall prevalence of COPD and bronchiectasis was $12.6 \%(95 \% \mathrm{CI}, 11.6-13.6 \%)$ and $0.8 \%(95 \%$ CI, $0.6-1.1 \%)$, respectively, in participants aged 40 or older. For subjects in their 40s, the prevalence of COPD and bronchiectasis was $4.2 \%$ and $0.2 \%$, respectively, For those in their $50 \mathrm{~s}$, the prevalence was $9.9 \%$ and $1.1 \%$, respectively; and for those aged 60 or older $24.8 \%$ and $1.2 \%$ had COPD and bronchiectasis (Figure 2). The average ages of subjects in the control, COPD, and bronchiectasis groups were 54.1, 64.0, and 59.0 years, respectively. Compared to other subjects, those with COPD were older on average (64.0 years, $\mathrm{P}<0.001$; Table 1 ). By sex, the proportion of men was highest in subjects with COPD, followed by those with bronchiectasis and those in the control group $(70.0 \%$, $52.4 \%$, and $44.8 \%$, respectively, $\mathrm{P}<0.001)$. The proportion of subjects with low family income was highest in subjects with bronchiectasis, followed by those with COPD and those in the control group $(77.9 \%, 63.5 \%$, and $43.7 \%$, respectively, $\mathrm{P}<0.001)$. Whereas control subjects were most likely to be manager/professional/office workers (26.0\%), COPD and bronchiectasis subjects were most likely to be agriculture/fishery workers $(28.2 \%)$ and service/sales 
Page 4 of 9

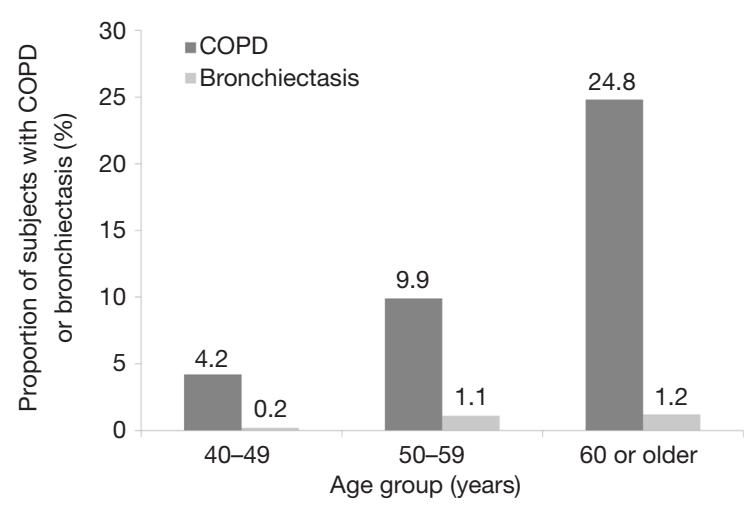

Figure 2 Prevalence of COPD and bronchiectasis by age. COPD, chronic obstructive pulmonary disease.

workers $(41.7 \%)$, respectively $(\mathrm{P}<0.001)$.

Regarding comorbidities, bronchiectasis subjects had the highest prevalence of asthma (18.0\%), previous history of pulmonary tuberculosis (40.5\%), and osteoporosis (19.1\%), while COPD participants had the highest prevalence of diabetes mellitus (17.6\%), hypertension (46.6\%), and dyslipidemia (49.7\%).

\section{Comparison of symptoms and quality of life}

As shown in Table 2, Subjects with bronchiectasis had similar degrees of respiratory symptoms such as cough (24.0\%, $\mathrm{P}=0.406)$ and sputum $(22.0 \%, \mathrm{P}=0.980)$, bad/ very bad perceived health status $(39.9 \%, \mathrm{P}=0.585)$, and limitation in physical activity due to respiratory diseases (34.8\%, $\mathrm{P}=0.066$ ) to COPD subjects, which were lower than control group $(\mathrm{P}<0.001$ for all respiratory symptoms above mentioned).

Quality of life, estimated by EQ-5D index, was lowest among subjects with bronchiectasis, followed by those with COPD and those in the control group $(0.87,0.89$, and 0.93, respectively, $\mathrm{P}<0.001)$. Compared to COPD subjects, bronchiectasis subjects were more likely to have limitation in usual activity $(28.8 \%$ vs. $20.4 \%, \mathrm{P}<0.001)$. However, there were no significant differences in the proportion of subjects with limitation in mobility $(\mathrm{P}=0.214)$ or pain/discomfort $(\mathrm{P}=0.059)$ between bronchiectasis and COPD groups.

\section{Associations of respiratory symptoms, physical activity, socioeconomic status, and quality of life with COPD or bronchiectasis versus control}

In univariable analysis, compared to the control group, respiratory symptoms (cough, sputum, or dyspnea) and limitation in physical activity were associated with COPD [unadjusted odds ratio $(\mathrm{OR})=2.06,95 \% \mathrm{CI}: 1.33-3.79$ for respiratory symptoms; unadjusted $\mathrm{OR}=5.96,95 \% \mathrm{CI}$ : $3.28-10.81$ for limitation in physical activity] and bronchiectasis (unadjusted OR $=7.40,95 \%$ CI: $1.79-30.59$ for respiratory symptoms; unadjusted $\mathrm{OR}=18.19,95 \% \mathrm{CI}$ : 5.23-63.28 for limitation in physical activity). However, in multivariable analysis, respiratory symptoms and limitation in physical activity were only associated with bronchiectasis (adjusted OR $=7.96,95 \%$ CI: 2.10-30.12 for respiratory symptoms; adjusted OR =9.43, 95\% CI: 1.06-83.79).

Although low education level (high school or less) was associated with COPD in univariable analysis (unadjusted OR $=1.96,95 \%$ CI: $1.46-2.65$ ), this was not significant in multivariable analysis (adjusted $\mathrm{OR}=1.22,95 \% \mathrm{CI}$ : $0.85-1.73)$. Low family income was associated with COPD (unadjusted OR $=2.24,95 \%$ CI: $1.85-2.72$ ) and bronchiectasis (adjusted OR $=4.54,95 \%$ CI: 2.14-9.63) in univariable analysis, but only income was significantly associated with bronchiectasis in multivariable analysis (adjusted OR $=3.61,95 \%$ CI: $1.75-7.47$ ). Low quality of life (EQ-5D <0.9) was associated with COPD in univariable analysis (unadjusted $\mathrm{OR}=1.85,95 \% \mathrm{CI}$ : $1.53-2.24$ ), but the association was not significant in multivariable analysis (adjusted OR =0.81, 95\% CI: 0.64-1.02) (Table 3).

\section{Discussion}

Using nationally representative data in Korea, the present study showed that the prevalence of physician-diagnosed bronchiectasis in subjects aged 40 years or older in Korea was $0.8 \%$. Compared to COPD subjects, bronchiectasis subjects were more likely to be younger, female, and never-smokers and have previous pulmonary tuberculosis and osteoporosis as comorbidities. As in COPD subjects, bronchiectasis subjects had low family income, and the proportion of subjects working as manager/professional/ office workers was lower compared to control subjects. Bronchiectasis subjects have similar degrees of respiratory symptoms such as cough and sputum, worse perceived health status, and limitation in physical activity due to respiratory diseases compared to those in the COPD group. In multivariable analysis, respiratory symptoms, limitation in physical activity, and low family income were significantly associated with bronchiectasis.

There is a wide variance in prevalence estimates of bronchiectasis according to study method (2-4,23-27). 
Table 1 Comparison of the study population

\begin{tabular}{|c|c|c|c|c|c|}
\hline Variable & Total $(\mathrm{N}=6,934)$ & Control $(n=5,978)$ & $\operatorname{COPD}^{a}(n=904)$ & Bronchiectasis $^{\mathrm{b}}(\mathrm{n}=52)$ & $P$ value \\
\hline Sex, male & $52.0(50.7-53.3)$ & $44.8(43.4-46.2)$ & $70.0(65.7-73.9)$ & $52.4(35.0-69.3)$ & $<0.001$ \\
\hline $\mathrm{BMI}, \mathrm{kg} / \mathrm{m}^{2}$ & $24.3(24.2-24.4)$ & $24.4(24.3-24.5)$ & $23.3(23.0-23.5)$ & 23.2(22.4-24.1) & $<0.001$ \\
\hline Smoking history & & & & & $<0.001$ \\
\hline Family income & & & & & $<0.001$ \\
\hline Low & $46.3(44.1-48.6)$ & $43.7(41.4-46.0)$ & $63.5(58.9-67.8)$ & $77.9(62.2-88.3)$ & \\
\hline High & $53.7(51.4-55.9)$ & $56.3(54.0-58.6)$ & $36.5(32.2-41.1)$ & 22.1(11.7-37.8) & \\
\hline Type of occupation & & & & & $<0.001$ \\
\hline Manager/professional/office worker & $24.6(22.4-26.8)$ & $26.0(23.7-28.5)$ & $13.4(9.9-18.0)$ & $6.4(1.8-19.9)$ & \\
\hline Service/sales worker & $23.9(22.1-25.9)$ & $25.1(23.2-27.2)$ & $12.7(9.1-17.4)$ & $41.7(18.5-69.3)$ & \\
\hline Agriculture/fishery worker & $14.4(12.0-17.3)$ & $12.7(10.4-15.3)$ & $28.2(22.8-34.3)$ & $26.9(12.2-49.4)$ & \\
\hline Skilled labor/machine operation & $20.0(18.1-21.9)$ & $20.1(18.3-22.1)$ & $19.7(15.2-25.1)$ & $26.0(21.2-31.5)$ & \\
\hline Manual laborer & $17.1(15.6-18.8)$ & $16.0(14.5-17.7)$ & $26.0(21.2-31.5)$ & $19.5(6.7-44.8)$ & \\
\hline \multicolumn{6}{|l|}{ Comorbidities } \\
\hline \multicolumn{6}{|l|}{ Pulmonary comorbidity } \\
\hline Dyslipidemia & $46.2(44.7-47.7)$ & $45.7(44.1-47.3)$ & $49.7(45.6-53.8)$ & $45.3(28.4-63.4)$ & 0.250 \\
\hline Cardiovascular disease & $4.3(3.7-4.9)$ & $4.0(3.5-4.7)$ & $6.0(4.5-7.8)$ & $5.1(1.2-18.6)$ & 0.064 \\
\hline Osteoporosis & $7.9(7.1-8.8)$ & $7.9(7.1-8.8)$ & $7.3(5.4-9.8)$ & $19.1(10.3-32.7)$ & 0.025 \\
\hline Osteoarthritis or rheumatoid arthritis & $17.9(16.7-19.1)$ & $17.4(16.2-18.7)$ & $20.3(16.6-24.6)$ & $30.8(17.6-48.1)$ & 0.061 \\
\hline Depression & $3.6(3.1-4.2)$ & $3.7(3.1-4.3)$ & $3.3(2.1-5.2)$ & $7.4(2.6-19.6)$ & 0.383 \\
\hline \multicolumn{6}{|l|}{ Spirometry } \\
\hline FVC, L & $3.5(3.4-3.5)$ & $3.5(3.4-3.5)$ & $3.4(3.3-3.5)$ & $3.2(3.0-3.5)$ & 0.151 \\
\hline FVC, \% predicted & $91.6(91.1-92.0)$ & $92.0(91.6-92.5)$ & $88.5(87.3-89.8)$ & $86.0(81.8-90.3)$ & $<0.001$ \\
\hline $\mathrm{FEV}_{1}, \mathrm{~L}$ & $2.69(2.66-2.71)$ & $2.76(2.74-2.79)$ & $2.17(2.11-2.24)$ & $2.32(2.05-2.59)$ & $<0.001$ \\
\hline $\mathrm{FEV}_{1}, \%$ predicted & $91.0(90.6-91.5)$ & 93.3 (92.9-93.7) & 75.9 (74.6-77.3) & 79.8 (73.1-86.5) & $<0.001$ \\
\hline $\mathrm{FEV}_{1} / \mathrm{FVC}$ ratio & 77.7 (99.5-78.0) & 79.9 (79.7-80.1) & $63.2(62.6-63.7)$ & 71.6 (66.9-76.3) & $<0.001$ \\
\hline
\end{tabular}

Data are presented as weighted mean (95\% confidence interval) or weighted percentage (95\% confidence interval). ${ }^{\text {, }}$, COPD was defined as pre-bronchodilator $\mathrm{FEV}_{1} / \mathrm{FVC}$; ${ }^{\mathrm{b}}$, twenty-nine subjects showed airflow limitation. COPD, chronic obstructive pulmonary disease; BMI, body mass index; FVC, forced vital capacity; $\mathrm{FEV}_{1}$, forced expiratory volume in 1 second. 
Table 2 Comparison of symptoms, physical activity, perceived health, and quality of life among groups

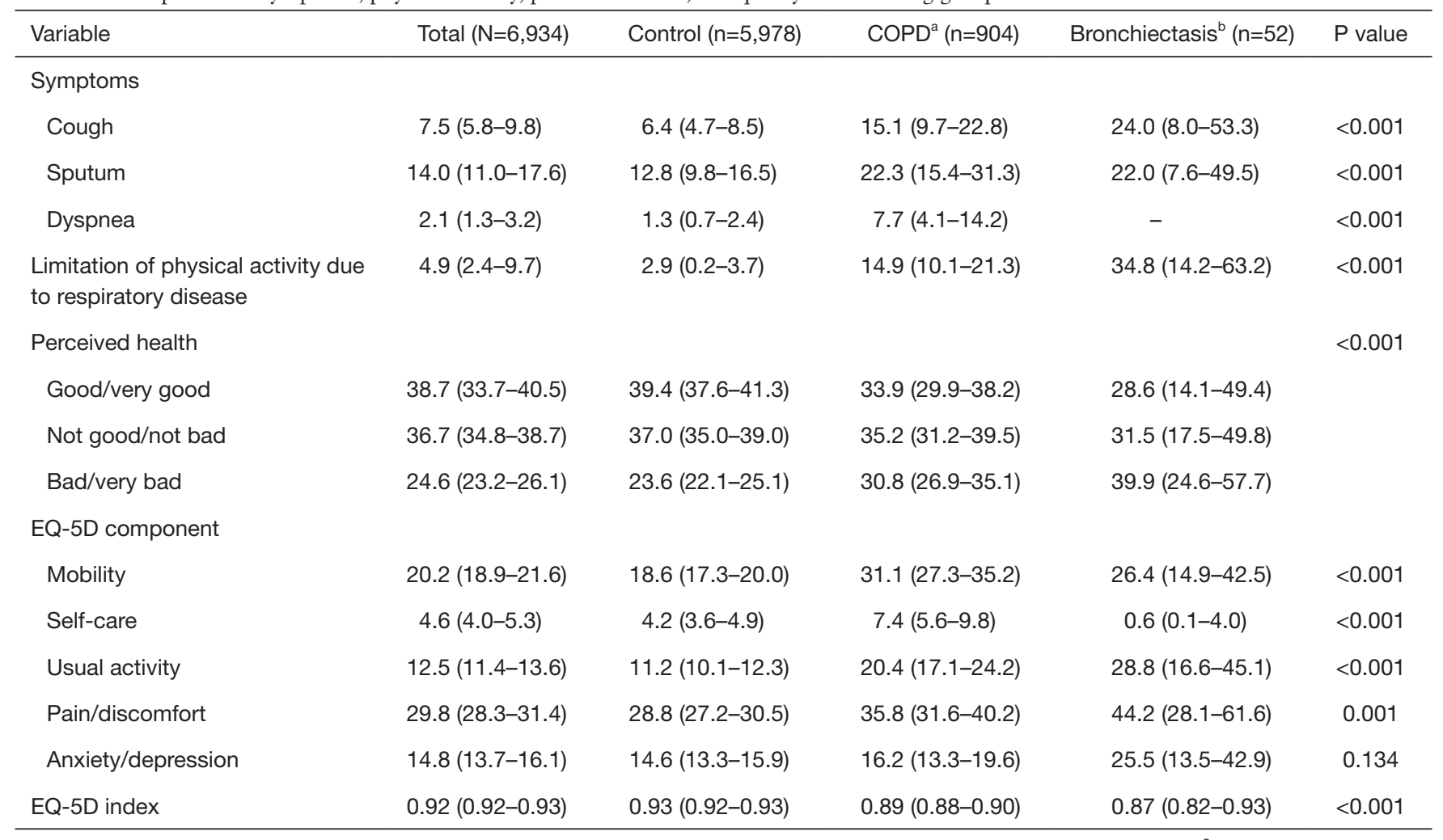

Data are presented as weighted mean (95\% confidence interval) or weighted percentage (95\% confidence interval). a , COPD was defined as pre-bronchodilator FEV1/FVC; ${ }^{\text {}}$, twenty-nine subjects showed airflow limitation. COPD, chronic obstructive pulmonary disease; EQ-5D, EuroQoL five dimensions.

In this study, the prevalence of physician-diagnosed bronchiectasis in subjects aged 40 years or older in Korea was $0.8 \%$, which is comparable to our previous study (8). In line with this result, a previous study reported that the prevalence of bronchiectasis in subjects aged 40 years or older in China was $1.2 \%$ in an urban population-based study (7). However, these prevalence rates are somewhat lower than the prevalence in a recent study in Korea, which reported that about $5 \%$ of patients who underwent chest tomography had bronchiectasis and respiratory symptoms (25). Accordingly, these results indicate that there may be under-recognition of this lung disease in real-world practice in Asia. These results also suggest that clinicians need to be mindful of the possibility of bronchiectasis in subjects with chronic respiratory symptoms when no other etiology can be identified.

The prevalence of bronchiectasis in Asians was suggested to be higher than that of Westerners $(4,7,8,25,27)$. However, there have been limited data to support this view; thus, our study provided informative data about the prevalence of bronchiectasis in Korea. The reasons for the different prevalences among Asian and Western populations have been suggested to be a higher prevalence of pulmonary infection and pulmonary tuberculosis in Asians (28-30), which is supported by a high prevalence of the previous history of tuberculosis in this study. However, to elucidate the reasons for the higher prevalence of bronchiectasis in the Asian population, further studies evaluating the etiologies of bronchiectasis in various Asian countries are needed. These studies should consider factors such as genetic susceptibility, environment, and socioeconomic status.

Our study showed that low socioeconomic status was associated with bronchiectasis. Compared to the control group, low family income was significantly associated with bronchiectasis. In addition, the proportion of subjects working as manager/professional/office workers was lowest in bronchiectasis subjects, while the proportion of subjects working as service/sales workers and skilled labor/machine operators was highest. Although low socioeconomic status is suggested to be linked to many chronic lung diseases, 
Table 3 Association of respiratory symptoms, physical activity, quality of life, and socioeconomic status with COPD or bronchiectasis versus control

\begin{tabular}{|c|c|c|c|}
\hline Variable & Control & $\mathrm{COPD}^{\mathrm{a}}$ & Bronchiectasis \\
\hline Univariable analysis & Reference & $2.06(1.33-3.79)$ & $7.40(1.79-30.59)$ \\
\hline Multivariable analysis & Reference & $1.62(0.92-2.85)$ & $7.96(2.10-30.12)$ \\
\hline \multicolumn{4}{|c|}{ Limitation of physical activity due to respiratory disease } \\
\hline Multivariable analysis & Reference & $1.84(0.64-5.27)$ & $9.43(1.06-83.79)$ \\
\hline \multicolumn{4}{|c|}{ Education level $\leq$ high school } \\
\hline Univariable analysis & Reference & $1.96(1.46-2.65)$ & $2.52(0.75-8.46)$ \\
\hline Multivariable analysis & Reference & $1.22(0.85-1.73)$ & $1.14(0.38-3.43)$ \\
\hline Multivariable analysis & Reference & $1.08(0.86-1.37)$ & $3.61(1.75-7.47)$ \\
\hline \multicolumn{4}{|l|}{$E Q-5 D<0.9$} \\
\hline Univariable analysis & Reference & $1.85(1.53-2.24)$ & $1.69(0.85-3.37)$ \\
\hline Multivariable analysis & Reference & $0.81(0.64-1.02)$ & $0.72(0.35-1.47)$ \\
\hline
\end{tabular}

Data are presented as odds ratio (95\% confidence interval). Multivariable analysis adjusted for age, sex, BMI, smoking status, education level, family income, asthma, tuberculosis, and FEV ${ }_{1}{ }^{\text {a }}$, COPD was defined as pre-bronchodilator FEV 1 /FVC. COPD, chronic obstructive pulmonary disease; BMI, body mass index; $\mathrm{FEV}_{1}$, forced expiratory volume in 1 second; EQ-5D, EuroQoL five dimensions.

there remain limited data on non-CF bronchiectasis (31). In this view, our study provided informative data that establish the association between socioeconomic status and bronchiectasis. However, since our study is cross-sectional and in disagreement with a previous study (2), future studies are needed to elucidate whether low socioeconomic status is a risk factor for the development of bronchiectasis.

Several studies revealed a close association between bronchiectasis and COPD $(32,33)$ or asthma (34-36). COPD or asthma subjects with bronchiectasis have a higher symptomatic burden and frequent exacerbation profile compared to those with COPD or asthma only (32-36). Although the causal inference between those diseases and bronchiectasis cannot be postulated by this study, our study showed that the presence of these airway diseases is closely associated with bronchiectasis, which is in agreement with previous studies. As shown in a study performed in China (7), previous pulmonary tuberculosis was one of the most common comorbidities in subjects with bronchiectasis. However, in Western populations, pulmonary tuberculosis was not common comorbidity $(14,37)$. Therefore, for management of comorbidities of bronchiectasis, ethnic or regional variance needs to be considered.

Interestingly, while the respiratory comorbid profile is similar between bronchiectasis and COPD subjects, there was variance in types of extra-pulmonary comorbidities between subjects with COPD and those with bronchiectasis. Whereas DM and hypertension were more frequent among COPD subjects, osteoporosis was more frequent among bronchiectasis subjects. Depression was more frequent in bronchiectasis subjects, but the difference was not statistically significant, probably due to relatively low prevalence. In agreement with our study results, previous studies showed that osteoporosis is frequently observed in subjects with bronchiectasis (37).

Many cohort studies demonstrated that the quality of life in subjects with bronchiectasis is significantly decreased (11-13). However, studies comparing the quality of life of bronchiectasis with other pulmonary diseases are rare. Overcoming this limitation by evaluating the quality of life in bronchiectasis subjects with COPD and/ or control subjects, our study showed that quality of life 
in bronchiectasis subjects measured by EQ-5D index was decreased, similar to that of COPD subjects. The burden of respiratory symptoms and limitation on physical activity due to respiratory disease were comparable to those of COPD subjects. This information may be helpful to clinicians; efforts to improve the quality of life of bronchiectasis patients are needed.

There are several limitations to this study. First, this study was performed in a representative sample of Korea. Thus, our data might not be generalizable to other ethnic groups or populations. Second, due to the cross-sectional design, we could not confirm a causal inference of the factors associated with bronchiectasis. Third, bronchiectasis was based on physician diagnosis. Therefore, more symptomatic subjects may have been included in this study, and subjects with minor symptoms may not have been included. Fourth, given the nature of Korea NHANES data, this study could not provide some clinical information related to respiratory symptoms and quality of life, which includes nontuberculous mycobacterial infection or home oxygen therapy. Fifth, the number of subjects with bronchiectasis was relatively small, which may have led to a lack of statistical significance. For example, bronchiectasis subjects are known to be at risk of cardiovascular diseases (38), however, there was no significant difference in the prevalence of cardiovascular diseases in this study.

In conclusion, the prevalence of bronchiectasis in subjects at least 40 years of age was $0.8 \%$ in Korea. The disease burden of bronchiectasis including respiratory symptoms, comorbidities, and quality of life was substantial and comparable to those of COPD subjects. However, previous tuberculosis and osteoporosis were more common in bronchiectasis subjects than in COPD subjects. Respiratory symptoms, limitation in physical activity, and low family income were significantly associated with bronchiectasis.

\section{Acknowledgments}

Funding: This research was supported by Basic Science Research Program through the National Research Foundation of Korea (NRF) funded by the Ministry of Science, Information and Communications Technologies (NRF-2019R1G1A1008692 to H Choi).

\section{Footnote}

Conflicts of Interest: The authors have no conflicts of interest to declare.
Ethical Statement: The authors are accountable for all aspects of the work in ensuring that questions related to the accuracy or integrity of any part of the work are appropriately investigated and resolved. Since publicly available data were used, our institution waived ethical approval. The Korea NHANES surveys were approved by the relevant institutional review boards; and written, informed consent was provided by all participants.

\section{References}

1. Polverino E, Goeminne PC, McDonnell MJ, et al. European Respiratory Society guidelines for the management of adult bronchiectasis. Eur Respir J 2017. doi: 10.1183/13993003.00629-2017.

2. Quint JK, Millett ER, Joshi M, et al. Changes in the incidence, prevalence and mortality of bronchiectasis in the UK from 2004 to 2013: a population-based cohort study. Eur Respir J 2016;47:186-93.

3. Weycker D, Hansen GL, Seifer FD. Prevalence and incidence of noncystic fibrosis bronchiectasis among US adults in 2013. Chron Respir Dis 2017;14:377-84.

4. Seitz AE, Olivier KN, Adjemian J, et al. Trends in bronchiectasis among medicare beneficiaries in the United States, 2000 to 2007. Chest 2012;142:432-9.

5. Diel R, Chalmers JD, Rabe KF, et al. Economic burden of bronchiectasis in Germany. Eur Respir J 2019. doi: 10.1183/13993003.02033-2018.

6. Chandrasekaran R, Mac Aogain M, Chalmers JD, et al. Geographic variation in the aetiology, epidemiology and microbiology of bronchiectasis. BMC Pulm Med 2018;18:83.

7. Lin JL, Xu JF, Qu JM. Bronchiectasis in China. Ann Am Thorac Soc 2016;13:609-16.

8. Choi H, Yang B, Nam H, et al. Population-based prevalence of bronchiectasis and associated comorbidities in South Korea. Eur Respir J 2019;54:1900194.

9. Jose A, Ramos TM, de Castro RAS, et al. Reduced Physical Activity With Bronchiectasis. Respir Care 2018;63:1498-505.

10. Bradley JM, Wilson JJ, Hayes K, et al. Sedentary behaviour and physical activity in bronchiectasis: a crosssectional study. BMC Pulm Med 2015;15:61.

11. Chalmers JD, Goeminne P, Aliberti S, et al. The bronchiectasis severity index. An international derivation and validation study. Am J Respir Crit Care Med 2014;189:576-85.

12. Quittner AL, O'Donnell AE, Salathe MA, et al. Quality of Life Questionnaire-Bronchiectasis: final psychometric analyses and determination of minimal important 
difference scores. Thorax 2015;70:12-20.

13. Lee BY, Lee S, Lee JS, et al. Validity and Reliability of CAT and Dyspnea-12 in Bronchiectasis and Tuberculous Destroyed Lung. Tuberc Respir Dis (Seoul) 2012;72:467-74.

14. McDonnell MJ, Aliberti S, Goeminne PC, et al. Comorbidities and the risk of mortality in patients with bronchiectasis: an international multicentre cohort study. Lancet Respir Med 2016;4:969-79.

15. Miller MR, Hankinson J, Brusasco V, et al. Standardisation of spirometry. Eur Respir J 2005;26:319-38.

16. Choi JK, Paek D, Lee JO. Normal predictive values of spirometry in Korean population. Tuberc Respir Dis (Seoul) 2005;58:230-42.

17. Korean Standard Statistical Classification, Statistics Korea (KOSTAT). Statistics Korea. 2007. Available online: https://kssc.kostat.go.kr:8443/ksscNew_web/index.jsp

18. Yu JI, Lim DH, Lee J, et al. Necessity of adjuvant concurrent chemo-radiotherapy in D2-resected LNpositive gastric cancer. Radiother Oncol 2018;129:306-12.

19. Mancia G, De Backer G, Dominiczak A, et al. 2007 Guidelines for the Management of Arterial Hypertension: The Task Force for the Management of Arterial Hypertension of the European Society of Hypertension (ESH) and of the European Society of Cardiology (ESC). J Hypertens 2007;25:1105-87.

20. Force USPST, Bibbins-Domingo K, Grossman DC, et al. Statin Use for the Primary Prevention of Cardiovascular Disease in Adults: US Preventive Services Task Force Recommendation Statement. JAMA 2016;316:1997-2007.

21. Lee H, Shin SH, Gu S, et al. Racial differences in comorbidity profile among patients with chronic obstructive pulmonary disease. BMC Med 2018;16:178.

22. American Diabetes A. Diagnosis and classification of diabetes mellitus. Diabetes Care 2005;28 Suppl 1:S37-42.

23. Chang AB, Bell SC, Torzillo PJ, et al. Chronic suppurative lung disease and bronchiectasis in children and adults in Australia and New Zealand Thoracic Society of Australia and New Zealand guidelines. Med J Aust 2015;202:130.

24. Twiss J, Metcalfe R, Edwards E, et al. New Zealand national incidence of bronchiectasis "too high" for a developed country. Arch Dis Child 2005;90:737-40.

25. Kwak HJ, Moon JY, Choi YW, et al. High prevalence of bronchiectasis in adults: analysis of CT findings in a health screening program. Tohoku J Exp Med 2010;222:237-42.

26. Chalmers JD, Sethi S. Raising awareness of bronchiectasis in primary care: overview of diagnosis and management strategies in adults. NPJ Prim Care Respir Med 2017;27:18.
27. Ringshausen FC, de Roux A, Diel R, et al. Bronchiectasis in Germany: a population-based estimation of disease prevalence. Eur Respir J 2015;46:1805-7.

28. Dhar R, Mohan M, D'souza G, et al. Phenotype characterization of non cystic fibrosis bronchiectasis in India: baseline data from an Indian Bronchiectasis Registry. Am J Respir Crit Care Med 2017;195:A4726.

29. Qi Q, Wang W, Li T, et al. Aetiology and clinical characteristics of patients with bronchiectasis in a Chinese $\mathrm{H}$ an population: A prospective study. Respirology 2015;20:917-24.

30. Guan WJ, Gao YH, Xu G, et al. Aetiology of bronchiectasis in Guangzhou, southern China. Respirology 2015;20:739-48.

31. Sahni S, Talwar A, Khanijo S, et al. Socioeconomic status and its relationship to chronic respiratory disease. Adv Respir Med 2017;85:97-108.

32. Martinez-Garcia MA, de la Rosa Carrillo D, SolerCataluna JJ, et al. Prognostic value of bronchiectasis in patients with moderate-to-severe chronic obstructive pulmonary disease. Am J Respir Crit Care Med 2013;187:823-31.

33. Mao B, Lu HW, Li MH, et al. The existence of bronchiectasis predicts worse prognosis in patients with COPD. Sci Rep 2015;5:10961.

34. Kang HR, Choi GS, Park SJ, et al. The effects of bronchiectasis on asthma exacerbation. Tuberc Respir Dis (Seoul) 2014;77:209-14.

35. Mao B, Yang JW, Lu HW, et al. Asthma and bronchiectasis exacerbation. Eur Respir J 2016;47:1680-6.

36. Padilla-Galo A, Olveira C, Fernandez de Rota-Garcia L, et al. Factors associated with bronchiectasis in patients with uncontrolled asthma; the NOPES score: a study in 398 patients. Respir Res 2018;19:43.

37. Gale NS, Bolton CE, Duckers JM, et al. Systemic comorbidities in bronchiectasis. Chron Respir Dis 2012;9:231-8.

38. Navaratnam V, Millett ER, Hurst JR, et al. Bronchiectasis and the risk of cardiovascular disease: a population-based study. Thorax 2017;72:161-6.

Cite this article as: Yang B, Choi H, Lim JH, Park HY, Kang D, Cho J, Lee JS, Lee SW, Oh YM, Moon JY, Kim SH, Kim TH, Sohn JW, Yoon HJ, Lee H. The disease burden of bronchiectasis in comparison with chronic obstructive pulmonary disease: a national database study in Korea. Ann Transl Med 2019;7(23):770. doi: 10.21037/atm.2019.11.55 